\title{
Relativistic effects in Kronig-Penney models on quasiperiodic lattices
}

\author{
Francisco Domínguez-Adame \\ Departamento de Fisica de Materiales, Facultad de Ciencias Fisicas, Universidad Complutense, E-28040 Madrid, Spain
}

and

\author{
Angel Sánchez \\ Departamento de Fisica Térica I, Facultad de Ciencias Fisicas, Universidad Complutense, E-28040 Madrid, Spain
}

Received 12 April 1991; accepted for publication 7 August 1991

Communicated by A.R. Bishop

\begin{abstract}
We study tunneling of Dirac particles through Kronig-Penney models on general lattices, computing their transmission cocfficient. We subsequently focus our attention on the Fibonacci lattice as a typical example of a quasicrystal. We compare our results to the non-relativistic ones, and find a shrinkage of the spectrum similar to that of periodic systems.
\end{abstract}

\section{Introduction}

In recent years, much attention has been paid to the study of aperiodic systems, either quasiperiodic or random. In particular, a large amount of work has been devoted to the one-dimensional (1D) Fibonacci chain, as a suitable model for quasicrystals [1-10] which in addition can be physically realized in the form of superlattices manufactured by molecular beam epitaxy [11]. This and other quasiperiodic systems are of great interest in solid state physics because they are structures being intermediate between periodic and fully disordered (random) ones. Electron dynamics in such models has been previously studied in the framework of the Schrödinger equation, i.e., from the viewpoint of non-relativistic quantum mechanics. However, it is well established that a proper treatment of the motion of electrons in condensed systems consisting of heavy atoms, whether crystalline or disordered, should take into account relativistic effects [12]. Relativistic electronic states have been considered in several works (see refs. [13-15], and references therein), in which the electron interaction with the crystal has been pre- sented by a $1 \mathrm{D}$ periodic array of $\delta$-function potentials. However, the study of relativistic electrons in disordered systems has received much less attention $[16,17]$, and, as far as we know, the motion of Dirac electrons in quasiperiodic lattices has been ignored in the literaturc.

In this Letter, we aim to study the tunneling of Dirac particles through quasiperiodic barriers. We choose the interaction potential to be

$V(x)=\lambda \sum_{j=1}^{N} \delta\left(x-x_{j}\right)$,

where we do not specify the positions $x_{j}$ at the moment. We first compute the transmission coefficient by means of the transfer matrix technique. We show that this coefficient can be cast in the form of a simple recurrence relationship that can be readily evaluated for any particular quasiperiodic or even random lattice. At a subsequent stage, we select the Fibonacci chain as a concrete cxample, in view of the broad current knowledge on electron transmission through it $[1-10]$. Instead of doing an exhaustive study of all of the electron propagation characteristics, we concern ourselves with several points 
trying to clarify what are the main new features arising from the inclusion of relativistic effects and different from the already known ones of non-relativistic systems.

\section{Single-site transfer matrix}

Let us consider the ID Dirac equation for a relativistic electron with energy $E$ and mass $m$, scattered by a single-site potential located at $x_{0}$, given by $V(x)=\lambda \delta\left(x-x_{0}\right)$, which, in the standard representation [18] and in units such that $\hbar=c=1$, reads

$\left[-\mathrm{i} \sigma_{x} \mathrm{~d} / \mathrm{d} x+\sigma_{z} m-E+\lambda \delta\left(x-x_{0}\right)\right] \psi(x)=0$,

with $\sigma_{x}$ and $\sigma_{z}$ being the $2 \times 2$ Pauli matrices and $\psi(x)$ the two-component wavefunction. In the force-free region, scattering solutions (i.e., solutions with $\left.E^{2}>m^{2}\right)$ of (2) can be written as

$$
\begin{aligned}
\psi(x) & =Q M(x) \phi_{+}, \quad \text { if } x>x_{0}, \\
& =Q M(x) \phi_{-}, \text {if } x<x_{0},
\end{aligned}
$$

where $\phi_{ \pm}$are two-component constant spinors and

$$
\begin{aligned}
& Q=\left(\begin{array}{cc}
1 & 1 \\
\xi^{-1} & -\xi^{-1}
\end{array}\right), \\
& M=\left(\begin{array}{cc}
\mathrm{e}^{\lg x} & 0 \\
0 & \mathrm{e}^{-\mathrm{i} q x}
\end{array}\right) .
\end{aligned}
$$

For the sake of simplicity, we have introduced the notations $q \equiv\left(E^{2}-m^{2}\right)^{1 / 2}$ and $\xi \equiv[(E+m) /$ $(E-m)]^{1 / 2}$, both $q$ and $\xi$ being real for $E^{2}>m^{2}$.

Our next step is to define the transfer matrix $T\left(x_{0}\right)$ for the single-site potential according to the relationship

$\phi_{+}=T\left(x_{0}\right) \phi_{-}$.

Taking into account that the boundary condition at $x=x_{0}$ for the relativistic (electrostatic-like) $\delta$-function potential is [19]

$$
\begin{aligned}
& \psi\left(x_{0}^{+}\right)=W(\lambda) \psi\left(x_{0}^{-}\right) \\
& \equiv\left(\begin{array}{cc}
\cos \lambda & -i \sin \lambda \\
-i \sin \lambda & \cos \lambda
\end{array}\right) \psi\left(x_{0}^{-}\right),
\end{aligned}
$$

and using (3) and (6) we find that the transfer matrix for a single-site potential is given by

$$
T\left(x_{0}\right)=M\left(-x_{0}\right) Q^{-1} W(\lambda) Q M\left(x_{0}\right) .
$$

This expression will now allow us to compute the transmission coefficient for the whole lattice in what follows.

\section{Transmission coefficient for the linear chain}

The transfer matrix $T_{N}$ for the potential $V(x)$ given in (1) is found as the product of the single-site matrices for each individual $\delta$-function potential,

$T_{N}=\prod_{j=N}^{1} T\left(x_{j}\right)$

For convenience, let us define the following unimodular matrices,

$P_{j} \equiv Q^{-1} W(\lambda) Q M\left(\Delta_{j}\right) \equiv\left(\begin{array}{cc}\alpha_{j} & \beta_{j} \\ \beta_{j}^{*} & \alpha_{j}^{*}\end{array}\right)$,

where $\Delta_{j} \equiv x_{j}-x_{j-1}$ and we have set $x_{0}=0$ without loss of generality. With this definition, some straightforward algebra yields for the matrix elements the following expressions,

$\alpha_{j}=[\cos \lambda-\mathrm{i}(E / q) \sin \lambda] \exp \left(\mathrm{i} q \Delta_{j}\right)$,

$\beta_{j}=-\mathrm{i}(m / q) \sin \lambda \exp \left(-\mathrm{i} q \Delta_{j}\right)$.

It can be simply checked that $\left|\alpha_{j}\right|^{2}-\left|\beta_{j}\right|^{2}=1$. Furthermore, notice that $\alpha_{j}$ and $\beta_{j}$ reduce, in the nonrelativistic and weak coupling limits, to those obtained by Würtz et al. [4] in their computation of the corresponding transfer matrix for the Schrödinger equation with the potential (1).

In terms of the matrices $P_{j}$, the total transfer matrix can be written, except by a constant phase factor, as follows,

$T_{N}=\prod_{j=N}^{1} P_{j}$

Recalling eq. (10), we can write

$T_{N}=\left(\begin{array}{ll}A_{N} & B_{N} \\ B_{N}^{*} & A_{N}^{*}\end{array}\right)$,

with $\left|A_{N}\right|^{2}-\left|B_{N}\right|^{2}=1$. Since $T_{N}=P_{N} T_{N-1}$, we can immediately derive the recurrence relationship 


$$
\begin{aligned}
A_{N} & =\left(\alpha_{N}+\alpha_{N-1}^{*} \frac{\beta_{N}}{\beta_{N-1}}\right) A_{N-1}-\frac{\beta_{N}}{\beta_{N-1}} A_{N-2}, \\
N & =2,3, \ldots,
\end{aligned}
$$

with initial conditions $A_{0}=1, A_{1}=\alpha_{1}$. If we call $R_{N} \equiv \operatorname{Re}\left(A_{N}\right)$ and $I_{N} \equiv \operatorname{Im}\left(A_{N}\right)$, eq. (15) splits into the following two expressions,

$$
\begin{aligned}
R_{N} & =2\left[\cos \lambda \cos \left(q \Delta_{N}\right)+(E / q) \sin \lambda \sin \left(q A_{N}\right)\right] R_{N-1} \\
& -\cos \left[q\left(\Delta_{N}-\Delta_{N-1}\right)\right] R_{N-2} \\
& -\sin \left[q\left(\Delta_{N}-\Delta_{N-1}\right)\right] I_{N-2}, \\
I_{N} & =2\left[\cos \lambda \cos \left(q \Delta_{N}\right)+(E / q) \sin \lambda \sin \left(q \Delta_{N}\right)\right] I_{N-1} \\
& -\cos \left[q\left(\Delta_{N}-\Delta_{N-1}\right)\right] I_{N-2} \\
& +\sin \left[q\left(\Delta_{N}-\Delta_{N-1}\right)\right] R_{N-2},
\end{aligned}
$$

where the initial values are now $R_{0}=1, I_{0}=0$, $R_{1}=\cos \lambda \cos \left(q \Delta_{1}\right)+(E / q) \sin \lambda \sin \left(q \Delta_{1}\right)$ and $I_{1}=$ $\cos \lambda \sin \left(q \Delta_{1}\right)-(E / q) \sin \lambda \cos \left(q \Delta_{1}\right)$.

As in the non-relativistic case, the transmission coefficient $\tau_{N}(E)$ for the linear chain can be calculated from the matrix element $A_{N}$ in the form

$\tau_{N}(E)=\frac{1}{\left|A_{N}\right|^{2}}=\frac{1}{R_{N}^{2}+I_{N}^{2}}$.

For each particular configuration of the lattice, $R_{N}$ and $I_{N}$ can be obtained recursively from eqs. (16), (17) and the transmission coefficient subsequently cvaluated from eq. (18). As far as we have not imposed any constraint concerning the positions of the $\delta$-functions, our treatment is valid for any lattice of equal strength scatterers, either periodic, quasi-periodic, or random. In the remaining of the paper, we will choose for definiteness the Fibonacci sequence to carry out some analysis of the differences between relativistic and non-relativistic transmission of particles through quasiperiodic systems.

\section{Results and discussion for the Fibonacci chain}

In order to facilitate a direct comparison to the non-relativistic treatment, let us define $\epsilon \equiv E / m-1$, which is nothing but the kinetic energy of the Dirac particle expressed in units of $m$. Besides, we measure lengths in units of $m^{-1}$ and take $\Delta_{1}=m^{-1}=1$. This does not restrict our computations because lengths and energies (also masses) appear always as quotients, and only their ratio will influence the transmission properties. Thus, with these conventions, we can now specify how our Fibonacci lattice is built up. We will fix the strengths of our $\delta$-function potentials to be $\lambda=\pi / 4$; the reason for this choice is simply to study the situation in which the trigonometric functions in the recurrence relationships (16), (17) contribute in the same amount to the transmission coefficient. On the other hand, the positions of the $\delta$ function potentials are specified by [3]

$x_{j}=j+(a-1)\left[\frac{j+1}{\omega_{0}}\right]$,

where $\omega_{0} \equiv(1+\sqrt{5}) / 2$ is the golden mean, $[z]$ stands for the integer number part of $z, z$ any real number, and $a>1$ is the lattice generator. In some sense, $a$ is a measure of the deviation of the lattice from perfect periodicity. Notice that the Fibonacci chain consists of alternating intervals of lengths 1 and $a$ arranged according to the Fibonacci sequence. So, the greater $a$ is the larger the difference between both intervals becomes, and the more different the system is from a regular lattice.

Let us now turn to the results. We have summarized the most interesting ones in figs. 1-3. Fig. 1 shows the relativistic and non-relativistic transmission coefficients as a function of the particle energy for a typical Fibonacci chain of order 16, i.e., with $N=F_{16}=987 \delta$-function potentials, and a lattice generator $a=2$. In both cases, we have observed the occurrence of energy gaps, for which transmission is actually negligible. Our numerical evaluation of the recurrence relationships (16), (17) for different values of the lattice generator has allowed us to establish that the number of gaps increases with increasing $a$, i.e., it increases as we get away from the periodic lattice. There are also a number of energy intervals or peaks for which the transmission coefficient is close to unity. As could be expected, the number of peaks increases (and their width decreases) with the number of scatterers $N$. Concerning the differences between relativistic and non-relativistic phenomena, we must conclude that the inclusion of relatively does not open any new energy gap; rather well, it causes the shrinkage of the whole 

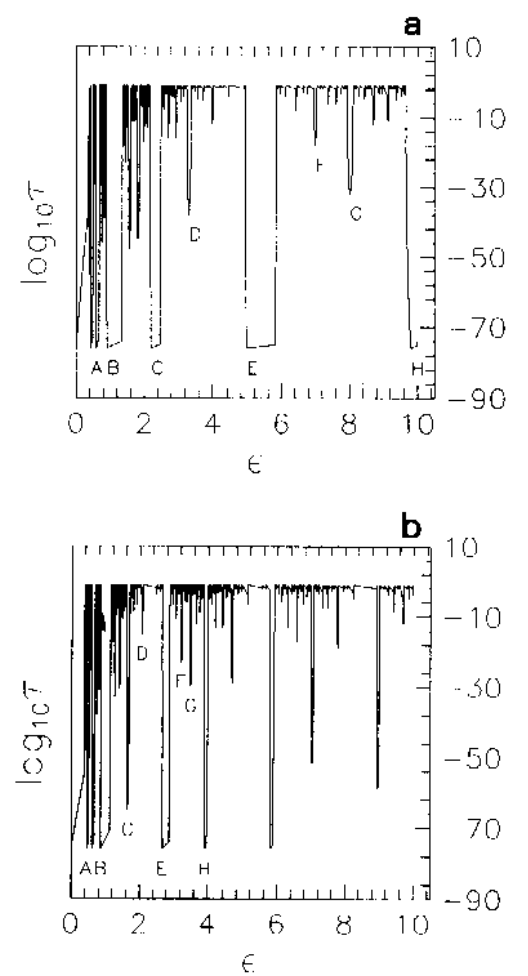

Fig. 1. (a) Non-relativistic and (b) relativistic transmission coefficients versus particle energy in a Fibonacci chain with $N=F_{16}=987$ scatterers and lattice generator $a=2$.

spectrum (check the lettering indicating the same gaps in both cases in fig. 1). To our understanding, this is a very interesting result, since the same behaviour has been found to occur in periodic systems [20]. Hence we can say that, with respect to transmission properties, periodic and quasiperiodic systems exhibit the same behaviour upon taking into account relativistic effects.

As a function of the chain length, the transmission coefficient shows an exponential decay for energy values belonging to gaps (see fig. 2). The corresponding decay (or localization) length does not strongly depend on the precise value of the energy provided it is actually in a gap. When a comparison is made for energies in the same gap, we found it to be a little larger in the relativistic case, that is to say, relativistic particles penetrate further in the chain. On the other hand, localization length diminishes with increasing $a$. This is not a surprising result because, as we have already mentioned, the use of large $a$ values amounts to building more disordered lat-
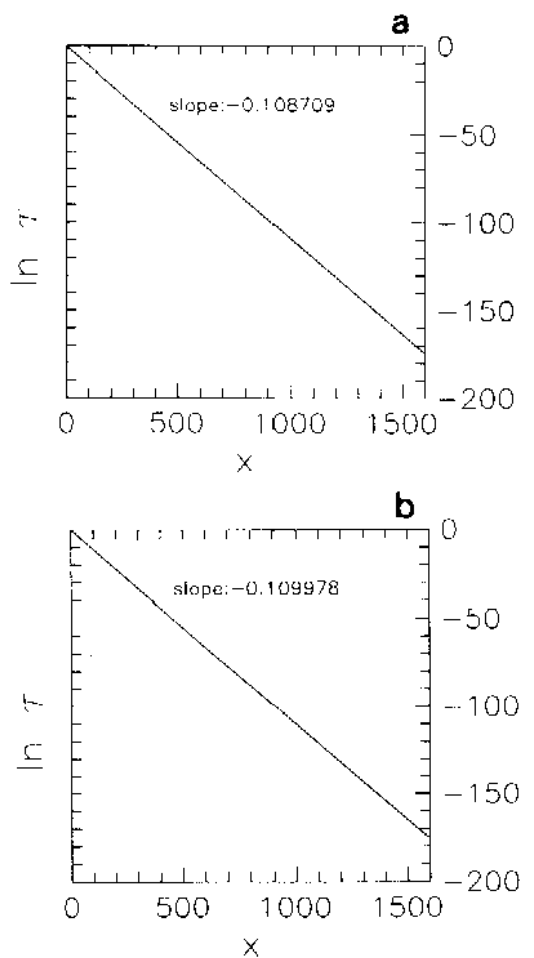

Fig. 2. Transmission coefficient versus chain length for energies in the gaps of the same lattice of fig. 1. (a) Non-rclativistic case, $\epsilon=4.966$ (at the bottom of the gap. $E$ of fig. 1). (b) Relativistic case, $\epsilon=2.672$ (also at the bottom of gap $E$ ).

tices, and this greater disorder gives rise to a stronger localization effect. However, we must say that the relativistic decay length diminishes faster with $a$ than the non-relativistic one, and for the value $a=2$ they are more or less the same.

The behaviour of the transmission coefficient as a function of the chain length becomes quite more complicated when the energy value considered is not one in a gap (see fig. 3). Both non-relativistic and relativistic particles show an approximately periodic dependence of their corresponding transmission coefficients on $N$, with variation ranges from values practically equal to unity to $10^{-5}$. The period of oscillations is larger the greater $a$ becomes. It is also larger for relativistic than for non-relativistic scattering. We stress again that comparisons must be carried out for energy values that are not numerically similar, but for those which correspond to states in a similar position in the spectrum, having in mind the shrinkage. This is so because we have found that the amplitude of the oscillations of the transmission 

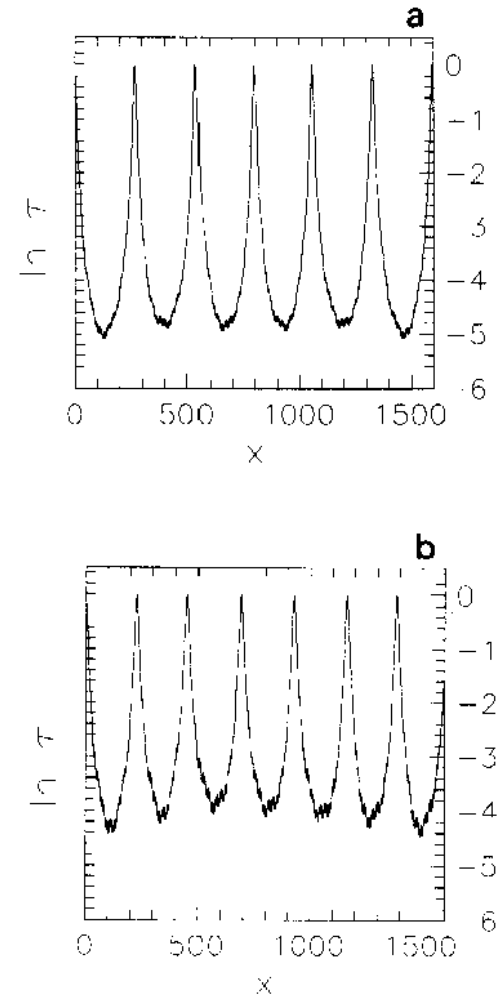

Fig. 3. Transmission coefficient versus chain length for energies in the allowed bands of the same lattice of fig. 1. (a) Non-relativistic casc, $\epsilon=5.846$ (right at the edge of gap $E$ ). (b) Relativistic case, $\epsilon=2.878$ (also at the edge of gap $E$ ).

coefficient depends on the position of the considered energy value in the allowed band. Allowed energies very close to a gap show oscillations in ranges much larger than energies well inside an allowed interval, both in the relativistic and in the non-relativistic transmission.

In summary, we have developed a formalism to study relativistic transmission through general lattices. As an illustration, we have applied it to a Fibonacci lattice of equal strength, $\delta$-function potentials. We have found that the main difference caused by relativity is the shrinkage of the spectrum. As a consequence, the spectrum of a fixed energy interval becomes more complex, with more gaps and allowed bands than in the non-relativistic problem. A remarkable feature of shrinkage is that, as allowed bands are narrower in the relativistic case, the os- cillations in the transmission coefficient as a function of the chain length are more pronounced, whereas for non-relativistic energies around the center of an allowed band those oscillations are small and the transmission coefficient is practically constant.

\section{Acknowledgement}

We want to thank C.L. Roy for calling our attention to some works on relativistic disordered systems. A.S. thanks L. Vázquez for encouragement, and the C.I.C.y T. (Spain) for partial financial support under project MAT90-0544.

\section{References}

[1] M. Kohmoto, L.P. Kadanoff and C. Tang, Phys. Rev. Lett. 50 (1983) 1870 .

[2] S. Ostlund, R. Pandit, D. Rand, H.J. Schellnhuber and E. Siggia, Phys. Rev. Lett. 50 (1983) 1873.

[3] J. Kollár and A. Sütö, Phys. Lett. A 117 (1986) 203

[4] D. Würtz, M.P. Soerensen and T. Schneider, Helv. Phys. Acta 61 (1988) 345.

[5] G. Gumbs and M.K. Ali, J. Phys. A 21 (1988) L517.

[6] M. Severin, M. Dulea and R. Riklund, I. Phys. Cond. Matter 1 (1989) 8851 .

[7] R.B. Capaz, B. Koiller and S.L.A. de Queiroz, Phys. Rev. B 42 (1990) 6402 .

[8] D. Kiang, T. Ochiai and S. Datć, Am. J. Phys. 58 (1990) 1200.

[9] Y. Liu and W. Sritrakool, Phys. Rev. B 43 (1991) 1110.

[10] M.E.J. Newman and R.B. Stinchcombe, Phys. Rev. B 43 (1991) 1183 .

[11] R. Merlin, K. Bajema, F.Y. Juang and P.K. Bhattacharya, Phys. Rev. Lett. 55 (1985) 1768.

[12] C.L. Roy, Physica B 113 (1982) 94.

[13] W.M. Fairbairn, M.L. Glasser and M. Steslicka, Surf. Sci. 36 ( 1973$) 462$.

[14] B.H.J. McKellar and G.J. Stephenson, Phys. Rev. C 35 (1987) 2262 .

[15] F. Dominguez-Adame, J. Phys. Cond. Matter 1 (1989) 109.

[16] C.L. Roy, Phys. Lett. A 118 (1986) 32.

[17] C.L. Roy, J. Phys. Chem. Solids 50 (1989) 11 1.

[18] F. Domínguez-Adame and M.A. González, Europhys. Lett. 13 (1990) 193.

[19] F. Domínguez-Adame and E. Maciá, J. Phys. A 22 (1989) L419.

[20] B. Méndez and F. Domínguez-Adame, J. Phys. A (1991) in press. 